\title{
Estimation of the total error of modal wavefront reconstruction with Zernike polynomials and Hartmann-Shack test
}

\author{
Oleg Soloviev $^{a}$ and Gleb Vdovin ${ }^{a, b}$ \\ ${ }^{a}$ EI Lab., EWI, TU Delft, Mekelweg 4, 2628CD Delft, the Netherlands \\ ${ }^{b}$ OKO Technologies B.V., P.O. Box 581, 2600AN Delft, the Netherlands
}

\begin{abstract}
The paper discusses the influence of the Hartmann-(Shack) wavefront sensor geometry on the total error of modal wavefront reconstruction. A mathematical model is proposed which describes modal wavefront reconstruction based on Hartmann or Hartmann-Shack sensor in terms of linear operators. The modal covers the most general case and is not limited by the orthogonality of decomposition basis or by the method chosen for decomposition. The total reconstruction error is calculated for any given statistics of the wavefront to be measured. Based on this estimate, total reconstruction error is calculated for regular and randomised Hartmann masks. The calculations demonstrate that use of random masks with non-regular Fourier spectra for Zernike wavefront reconstruction for atmospheric turbulence allows to double the number of decomposition modes with the same total error.
\end{abstract}

Keywords: wavefront sensing, Hartmann-Shack sensors, wavefront reconstruction, error minimisation

\section{INTRODUCTION}

Hartmann or Hartmann-Shack (HS) wavefront sensor ${ }^{1,2}$ is one of the most widely used in adaptive optics (AO) systems, where monochromatic light source and/or reference beam are often not available. The HS sensor measures the averaged local tilts over an array of sub-apertures (s/a) - holes in a Hartmann screen, or lenslets in HS test. From these local measurements, the wavefront can be reconstructed.

The problem of wavefront reconstruction with HS test has been addressed in many publications. ${ }^{3-16}$ There can be distinguished two general approaches, modal and zonal reconstruction. Zonal algorithms ${ }^{3-6,13}$ build discrete phase field with finite differences closest to the measured slopes in the least-square sense. The phase data in between the measured points are interpolated. Modal algorithms ${ }^{5,7,17}$ approximate the wavefront's phase by a linear combination of a number of aperture functions, or modes. According to Southwell, ${ }^{5}$ the modal approach is superior to zonal estimation in terms of error propagation, and is computationally easier and faster.

In adaptive optics systems, commonly modal algorithms are used, with different choice of decomposition modes and methods. Zernike polynomials are often chosen as decomposition basis due to their connection with classical aberrations; Karhunen-Loève functions or their pseudo-analytical analogues ${ }^{10}$ present an optimal basis for compensation of atmospheric turbulence; exponential functions are suitable for direct demodulations of hartmannograms ${ }^{14,15}$; influence functions of the adaptive element are used in any case to correct the aberrations.

The error of modal wavefront reconstruction was analysed by a number of authors. ${ }^{7,9,11,12,17}$ Herrmann ${ }^{17}$ introduced the basis gradient matrix and used it to describe cross-coupling and aliasing effects; Roggemann ${ }^{7}$ proposed to use minimum-variance reconstruction algorithm instead of standard least-square minimisation, which makes no use of wavefront's aberration statistics; Dai ${ }^{9,11}$ developed a clear theory to analyse the total modal reconstruction error and apply it to define the optimal number of decomposition modes for various sensor configuration.

In this paper, we address the modal reconstruction error using Dai's approach of total reconstruction error minimisation. Dai ${ }^{9,11}$ has shown that this error is difficult to eliminate and one thus should either define the optimal number of

For correspondence, please contact Oleg Soloviev

by e-mail (preferably) O.Soloviev@EWI.TUDelft.nl

or by phone: +31152786285

5th International Workshop on Adaptive Optics for Industry and Medicine, edited by Wenhan Jiang, Proc. of SPIE Vol. 6018, 60181D, (2005) · 0277-786X/05/\$15 · doi: 10.1117/12.669383 
decomposition modes for a given sensor geometry, or to use iterative methods to increase this number while keeping the error at the same level. However, iterative methods increase the computation time, a crucial factor in real-time AO systems. We propose here to use randomised sensor geometry, which doubles the number of correctly reconstructed modes, has the same computational time and smaller total reconstruction error. We also show, that the randomisation of the sensor geometry via small movements of s/a centres, although eliminates cross-talk of low-order aberrations, does not decrease the total error and should not be used.

Next section presents the most general description of the modal wavefront reconstruction in matrix, or linear operators notation. A formula for the total reconstruction error is derived in a more general, then that of Dai, ${ }^{11}$ context, which is valid also for not least-square algorithms. In the following sections, the theory is applied to build and compare Hartmann reconstruction matrices for five sensor geometries and least-square minimisation. Then, total reconstruction error is calculated for the case of Kolmogorov's atmosphere turbulence.

\section{MATHEMATICAL DESCRIPTION OF HS WAVEFRONT SENSING}

\subsection{HS sensor as a linear operator}

In modal wavefront sensing with Hartmann or Hartmann-Shack (HS) sensor, the incoming phase distributions $f=f(x, y)$ is represented by the sensor as some function $\tilde{f}$, which is a linear combination of some given decomposition modes $f_{j}, j=$ $1 \ldots, N$. Because this representation is based on linear operations of measurements of the averaged over subapertures wavefront slope and least-square approximation of this slopes, the Hartmann sensing mathematically can be represented as a linear operator $\mathcal{H}$, such that

$$
\tilde{f}=\mathcal{H} f=\sum_{j=1}^{N} \lambda^{j} f_{j}=\lambda^{j} f_{j},
$$

where $\lambda^{j}$ are the coefficients of the modal decomposition*. Due to linearity of $\mathcal{H}$, if the incoming wavefront can be decomposed over some "native" basis $g_{i}, i=1, \ldots, \infty$, (eg. Zernike polynomials for optical shop tests, K-L functions for $\mathrm{AO}$ in turbulent media), so one can write

$$
f=c^{i} g_{i}+c^{0},
$$

where the constant (piston) term $c^{0}$ is not sensed by HS-sensor, then the Hartmann operator $\mathcal{H}$ is fully defined by its matrix $\mathbf{H}=\left(h_{i}^{j}\right), i=1, \ldots, \infty, j=1, \ldots, N$. The elements $h_{i}^{j}$ of the matrix $\mathbf{H}$ are given by the Hartmann-images of the basis function:

$$
\mathcal{H} g_{i}=\sum_{j=1}^{N} h_{i}^{j} f_{j}
$$

and thus and one has

$$
\mathcal{H} f=\left(f_{1}, f_{2}, \ldots, f_{N}\right) \cdot \mathbf{H} \cdot\left(\begin{array}{l}
c^{1} \\
c^{2} \\
\ldots
\end{array}\right)=h_{i}^{j} c^{i} f_{j} .
$$

The properties of the HS wavefront sensor is thus defined by the properties of the matrix $\mathbf{H}$, which, in turn, depends on the sets of basis and decompositions functions $f_{j}$ and $g_{i}$, on the HS mask or lenslets geometry, and on the demodulation algorithm. These properties are discussed in following subsections, and first we dwell on common properties of linear operators mapping infinite-dimensional space in finite-dimensional.

*for brevity, we have adopted here tensor notation, namely upper index denotes the rows and the lower - the columns; thus $\lambda^{j}$ is a column vector, and $f_{j}-$ a row-vector. The same index repeating both as superscript and subscript denotes the summation by this index. Moreover, to further simplify the formulae, we will use indices spanning always the same range for given letter, for instance, $j, j_{1}, j_{2}$ running always values $1, \ldots, N ; i, i_{1}, i_{2}=1, \ldots, \infty, l=N+1, \ldots, \infty$, and so on. We also will use this for splitting sums and vectors, for instance, we will write $c^{i}=c^{j}+c^{l}$ and $\left\langle c^{i} c_{i}\right\rangle=\left\langle c^{j} c_{j}\right\rangle+\left\langle c^{l} c_{l}\right\rangle$. 


\subsection{The error of the operator $\mathcal{H}$}

For given basis and decomposition functions, one can identify the functions with their decomposition coefficients, and operator $\mathcal{H}$ may be regarded as mapping an infinite-long vector $c^{i}$ in $N$-dimensional vector $\lambda^{j}$. Then, according to a wellknown fact from the linear algebra, the dimension of the subspace of all vectors mapped to zero, Ker $\mathcal{H}$, is infinity. Hence, that for any HS sensor there exists an unlimited choice of aberrations sensed as zero by the sensor, and so the natural definition for the error $\varepsilon$ of the HS sensor as

$$
\varepsilon=\|\mathcal{H} f-f\|
$$

is useless without some imposed restrictions on the possible forms of $f$. Usually, these restrictions are expressed in a form of statical properties of the expansion coefficients $c^{i}$ of the sensed function. This can be either Kolmogorov statistics of the Zernike coefficients of the plane wavefront passed through the turbulent media, or some expected values of optical aberrations for given polishing process etc. These will be transformed by the operator $\mathcal{H}$ in the probability properties of $\varepsilon$. Namely, one has:

$$
\langle\varepsilon\rangle=\langle\|f-\tilde{f}\|\rangle=\|\langle f\rangle-\langle\tilde{f}\rangle\|=\left\|\left\langle c^{i}\right\rangle g_{i}-\left\langle\lambda^{j}\right\rangle f_{j}\right\|=\left\|\left\langle c^{i}\right\rangle g_{i}-\left\langle c^{i}\right\rangle h_{i}^{j} f_{j}\right\|,
$$

which in often case of $\left\langle c^{i}\right\rangle=0$ leads to $\langle\varepsilon\rangle=0$. In applications with non-zero expected values of the decomposition coefficients, as, for instance, in optical shop tests, equation (1) provides a formula for a systematic error of HS sensor.

To simplify the calculations of the second moment of $\varepsilon$, let us consider the case where decomposition modes are just the first $N$ basis functions

$$
f_{j}=g_{j}
$$

and let the basis be orthonormal for some $\operatorname{dot} \operatorname{product}(f, g)$,so

$$
\left(g_{i}, g^{i^{\prime}}\right)=\delta_{i}^{i^{\prime}},
$$

where $\delta_{i}^{i}$ is the Kronecker symbol. Then one can write

$$
\begin{aligned}
\left\langle\varepsilon^{2}\right\rangle=\langle(f-\tilde{f}, f-\tilde{f})\rangle=\langle(f, f)\rangle-2\langle(f, \tilde{f})\rangle+\langle(\tilde{f}, \tilde{f})\rangle=\left\langle c^{i_{1}}, c_{i 2}\right\rangle\left(g_{i_{1}}, g^{i_{2}}\right)-2\left\langle c^{i} \lambda_{j}\right\rangle\left(g_{i}, g^{j}\right)+\left\langle\lambda^{j_{1}} \lambda_{j_{2}}\right\rangle\left(g_{j_{1}}, g^{j_{2}}\right) \\
=\left\langle c^{i} c_{i}\right\rangle-2\left\langle c^{j} \lambda_{j}\right\rangle+\left\langle\lambda^{j} \lambda_{j}\right\rangle,
\end{aligned}
$$

and thus

$$
\left\langle\varepsilon^{2}\right\rangle=\left\langle c^{i} c_{i}\right\rangle-2\left\langle c^{j} c_{i}\right\rangle h_{j}^{i}+h_{i_{1}}^{j}\left\langle c^{i_{1}} c_{i_{2}}\right\rangle h_{j}^{i_{2}}
$$

Hence the variation $\sigma_{\varepsilon}^{2}=\left\langle\varepsilon^{2}\right\rangle-\varepsilon^{2}$ can be calculated, provided the matrix of cross-correlation $\left\langle c^{i_{1}} c_{i_{2}}\right\rangle$ and matrix $\mathbf{H}$ are known. Note, that formula (4) includes infinite summations and in practice should be approximated with a finite sum, or use explicit estimates for $h_{i}^{j}$ and $\left\langle c^{i_{1}} c_{i_{2}}\right\rangle$.

Let us now consider a case of "ideal" Hartmann matrix $\mathbf{H}$, given by

$$
h_{i}^{j}=\delta_{i}^{j} .
$$

The HS-sensor with such a matrix is an ideal low-order filter in the space of basis function, giving exact coefficients for the first $N$ modes and being insensitive to the modes of the higher order:

$$
\mathcal{H}\left(\sum_{i=1}^{\infty} c^{i} g_{i}\right)=\sum_{j=1}^{N} c^{j} g_{j}
$$

In this case $\lambda^{j}=c^{j}$ and from (1) one has

$$
\langle\varepsilon\rangle=\left\|\sum_{l=N+1}^{\infty} c^{l} g_{l}\right\|
$$

for the mean error; and from (3)

$$
\left\langle\varepsilon^{2}\right\rangle=\left\langle c^{i} c_{i}\right\rangle-\left\langle c^{j} \lambda_{j}\right\rangle=\left\langle c^{l} c_{l}\right\rangle=\sigma_{\text {tr }}^{2} .
$$


The last term represents a mean-square error due to infinite-series truncation, a minimum possible error for the orthonormal basis. This explains the name "ideal" for such a matrix.

In practice, however, the matrix $\mathbf{H}$ is often only an approximation to the ideal one, and the error increases. If first $N$ rows of $\mathbf{H}$ form an identity matrix and other rows are not zeroes, then equation (5) is valid only for $i \leq N$

$$
h_{j}^{j^{\prime}}=\delta_{j}^{j^{\prime}}
$$

Using this, after splitting sums in index $i$ into two sums with indexes $j$ and $l$, one obtains from (4)

$$
\begin{aligned}
\left\langle\varepsilon^{2}\right\rangle & =\left\langle c^{j} c_{j}\right\rangle+\left\langle c^{l} c_{l}\right\rangle-2\left\langle c^{j} c_{j^{\prime}}\right\rangle h_{j}^{j^{\prime}}-2\left\langle c^{j} c_{l}\right\rangle h_{j}^{l}+h_{j_{1}}^{j}\left\langle c^{j_{1}} c_{j_{2}}\right\rangle h_{j}^{j_{2}}+h_{l_{1}}^{j}\left\langle c^{l_{1}} c_{j_{2}}\right\rangle h_{j}^{j_{2}}+h_{j_{1}}^{j}\left\langle c^{j_{1}} c_{l_{2}}\right\rangle h_{j}^{l_{2}}+h_{l_{1}}^{j}\left\langle c^{l_{1}} c_{l_{2}}\right\rangle h_{j}^{l_{2}} \\
& =\left\langle c^{j} c_{j}\right\rangle+\left\langle c^{l} c_{l}\right\rangle-2\left\langle c^{j} c_{j}\right\rangle-2\left\langle c^{j} c_{l}\right\rangle h_{j}^{l}+\left\langle c^{j} c_{j}\right\rangle+h_{l}^{j}\left\langle c^{l} c_{j}\right\rangle+\left\langle c^{j} c_{l}\right\rangle h_{j}^{l}+h_{l_{1}}^{j}\left\langle c^{l_{1}} c_{l_{2}}\right\rangle h_{j}^{l_{2}} \\
& =\left\langle c^{l} c_{l}\right\rangle+h_{l_{1}}^{j}\left\langle c^{l_{1}} c_{l_{2}}\right\rangle h_{j}^{l_{2}} .
\end{aligned}
$$

Now the error is given by the infinite-series truncation $\sigma_{\text {tr }}^{2}$ plus a new term due to aliasing of the high-order modes,

$$
\sigma_{\mathrm{al}}^{2}=h_{l_{1}}^{j}\left\langle c^{l_{1}} c_{l_{2}}\right\rangle h_{j}^{l_{2}}
$$

an aliasing error. The obtained expression coincides with that of Dai. ${ }^{11}$

It is obvious now, how important are properties of the $\mathbf{H}$ for the error estimation. Next sections consider the practical way to calculate the matrix.

\subsection{Dependence of the error on the sensor geometry}

To build the matrix $\mathbf{H}$, let us first try to separate the dependence on the geometry of the mask. Note, that the measuring of the averaged wavefront slopes in HS-sensing also represents a linear operator. Indeed, if the slopes are measured over $m$ subapertures $a_{k}, k=1, \ldots, m$, then hartmannogram contains encoded information about $m$ pairs of numbers

$$
\frac{1}{S_{a_{k}}}\left(\int_{a_{k}} \frac{\partial f}{\partial x} d \mathbf{r}, \int_{a_{k}} \frac{\partial f}{\partial y} d \mathbf{r}\right),
$$

where $S_{a_{k}}$ is the subaperture's area. If all subapertures have the same symmetrical form, thus that all $a_{k}$ 's characteristic functions $\chi_{a_{k}}$ are obtained from the same even function $\chi_{a}(\mathbf{r})$ :

$$
\chi_{a_{k}}(\mathbf{r})=\chi_{a}\left(\mathbf{r}-\mathbf{r}_{k}\right)
$$

where $\mathbf{r}_{i}$ are the subapertures' centres, these $m$ pairs can be presented as sampling of "filtered" function written as

$$
\frac{1}{S_{a}} \int_{\mathbb{R}^{2}} \chi_{a}\left(\mathbf{r}-\mathbf{r}_{k}\right) \nabla f(\mathbf{r}) d \mathbf{r}=\frac{1}{S_{a}} \chi_{a} * \nabla f
$$

in points $\mathbf{r}_{k}$. This is equivalent to multiplying the filtered wavefront by the sampling function

$$
\sum_{i=1}^{m} \delta\left(\mathbf{r}-\mathbf{r}_{k}\right)
$$

Thus the result of HS-sampling can be presented as a linear operator $\mathcal{G}$ such that

$$
\mathcal{G} f=\frac{1}{S_{a}} \sum_{k=1}^{m} \delta\left(\mathbf{r}-\mathbf{r}_{k}\right)\left(\chi_{a} * \nabla f\right) .
$$

Although $\mathcal{G} f$ consists of $m$ pairs of numbers, we will represent it as a $2 m$-vector for simplicity. Then in basis $g_{i}$ operator $\mathcal{G}$ is described by its matrix $\mathbf{G}$, formed by $\mathcal{G} g_{i}$ as $i$-th row. This "filtered basis' gradient" matrix contains an 


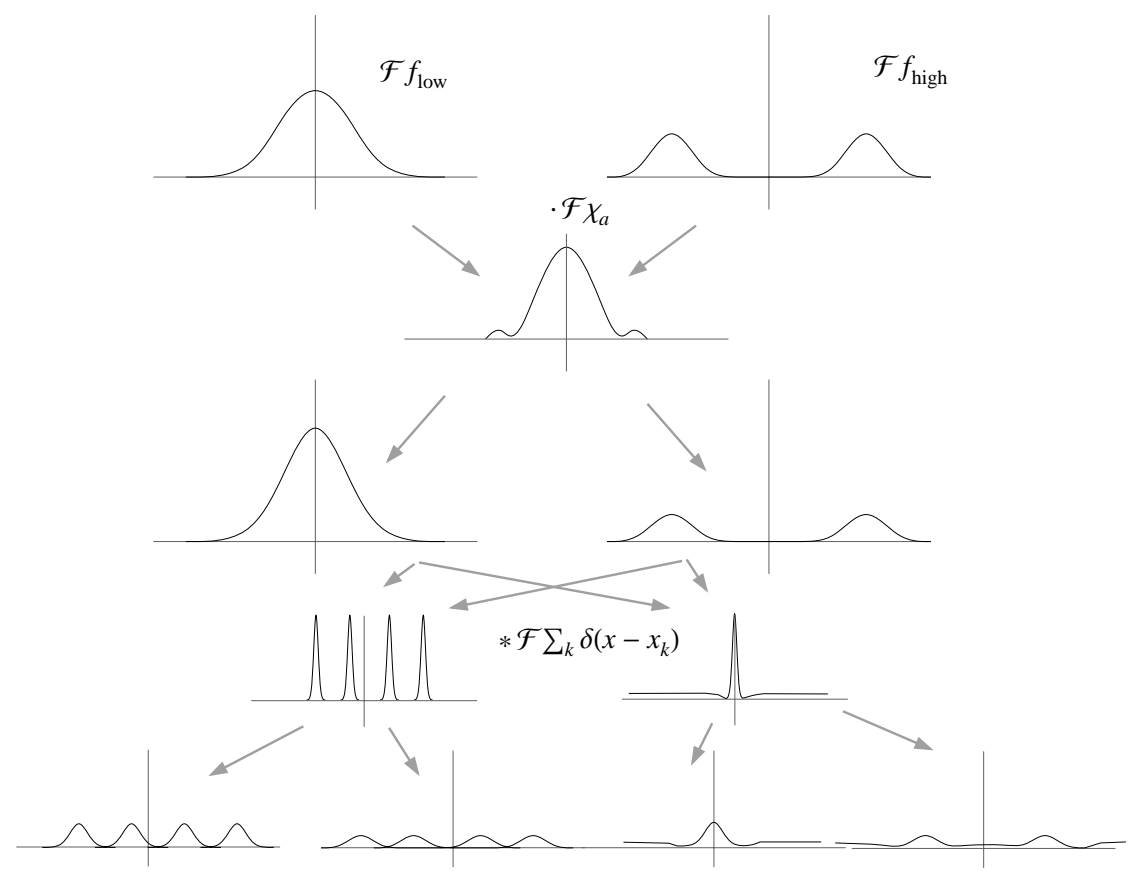

Figure 1: A schematic sketch of sensing high- and low-order modes for regular nd irregular sensor geometry. Note the similarity of sensed spectra in case of regular sampling (aliasing). Irregular geometry should not have aliasing effect.

important information about "compatibility" of the mask geometry and the basis functions. For instance, for regular 91holes hexagonal mask and Zernike polynomials as the basis, only first 87 rows of $\mathbf{G}$ are linearly independent. This means that for any further linear transformation, the dimension of the space of all reconstructed wavefronts will be less then 87 , and in general case the following inequality holds:

$$
\operatorname{dim} \operatorname{Im} \mathcal{H} \leq \operatorname{rank} \mathcal{G}
$$

Hence the number of possible reconstruction modes is limited by the rank of $\mathbf{G}$. Thus the geometries which result in matrices $\mathbf{G}$ with higher rank are desirable.

It seems to be extremely difficult to get an analytical dependence of rank $\mathbf{G}$ on the mask geometry. However, some insight can be obtained from the equation (10) considered in the frequency domain:

$$
\mathcal{F}(\mathcal{G} f)=\frac{1}{S_{a}} \mathcal{F}\left(\sum_{k=1}^{m} \delta\left(\mathbf{r}-\mathbf{r}_{k}\right)\right) *\left(\mathcal{F} \chi_{a} \cdot \mathcal{F} \nabla f\right)
$$

In this formula, the influences of subaperture size and centres are separated. If, as usual, the higher order modes contain higher frequencies, the term $\mathcal{F} \chi_{a} \cdot \mathcal{F} \nabla f$ leads to sensing of high frequency modes as low-frequency ones, because $\mathcal{F} \chi_{a}$ is a low-pass filter. On the another hand, the term $\mathcal{F}\left(\sum_{k=1}^{m} \delta\left(\mathbf{r}-\mathbf{r}_{k}\right)\right)$, given by the centres position, broaden the spectrum of the low-frequency functions. For regular masks, this broadening is performed in a regular way, and the resulting spectrum is similar to spectrum of high-order mode, also regular due to the symmetry. As a result of this operation, some different modes can be seen by the sensor as very similar (see Figure 1 for illustration).This, in turn, produces ill-defined G. An irregular centres distribution does not have a periodical spectrum and should increase the rank of the matrix $\mathbf{G}$.

\subsection{Dependence on the decomposition functions}

The matrix $\mathbf{H}$ is defined not only by the gradients matrix $\mathbf{G}$, but also by the choice of the decomposition functions $f_{j}$, or, more accurately, by the choice of the decomposition vectors $\hat{f}_{j}$ generated by $f_{j}$. The possible choices of $f_{j}$ are: 
- the same basis function $g_{j}$, e.g. Zernike polynomials;

- the influence, or response functions $r_{j}$ of the adaptive optical element;

- an arbitrary set of functions $f_{j}$ satisfying the desired properties, e.g. trigonometrical basis appearing in the Fourier analysis of the hartmannograms.

The $2 m$-vectors $\hat{f}_{j}$ are obtained from the chosen $f_{j}$ either by applying the same gradient operator $\mathcal{G}$,

$$
\hat{f}_{j}=\mathcal{G} f_{j},
$$

or, sometimes, by sampling unfiltered (one-point gradients) ${ }^{17}$ (or filtered with another filter) gradients of the decomposition functions

$$
\hat{f}_{j}=\sum_{k=1}^{m} \delta\left(\mathbf{r}-\mathbf{r}_{k}\right) \nabla f_{j} .
$$

In any case, the coefficients $\lambda^{j}$ are found as the closest by some norm $\|\bullet\|$ vector in space generated by $\hat{f}_{j}$ :

$$
\left\|\mathcal{G}-\lambda^{j} \hat{f}_{j}\right\|=\min _{x^{1}, \ldots, x^{N}}\left\|\mathcal{G}-x^{j} \hat{f}_{j}\right\| .
$$

Independently from the choice of $f_{j}, \hat{f}_{j}$, $\|\bullet\|$, the result of the minimisation can be presented in the form of some linear operator $\mathcal{L}$ :

$$
\lambda^{j}=\mathcal{L}(\mathcal{G} f)=(\mathcal{L} \circ \mathcal{G}) f=\mathcal{H} f
$$

and thus

$$
\mathbf{H}=\mathbf{L} \cdot \mathbf{G} \text {. }
$$

From this equation, it is obvious that to minimise the HS-sensor error, one must to chose proper matrix $\mathbf{L}$, and not the $\hat{f}_{j}$ or $\|\bullet\|$, which can be chosen a posteriori. For instance, if the decomposition modes coincide with the basis function, one usually uses a pseudoinverse for the first $N$ rows of $\mathbf{G}$ (denoted as $\mathbf{G}_{N}$ ) as matrix $\mathbf{L}$

$$
\mathbf{L}=\mathbf{G}_{N}^{+} \text {, }
$$

to obtain first $N$ rows of $\mathbf{H}$ closer to the identity matrix, and to eliminate the systematic error. If the systematic error is expected to be zero, the choice of $\mathbf{L}$ given by the equation (11) may not necessary provide the minimum variance of the error. The discussion of this topic however is beyond the scope of the present paper, and further we restrict ourselves to the case of equation (11).

\subsection{Gradient measurement and jittering error}

There exist two additional sources of the error in HS wavefront sensing. The first is the measurement error of the spots centers in the hartmannogram, $e^{k}$, say, so one has

$$
\lambda^{j}=\mathcal{L}\left(\mathcal{G} f+e^{k}\right)=\mathcal{H} f+\mathcal{L} e^{k} .
$$

If the measurement errors are zero-mean independent variables, uncorrelated with coefficients $c^{i}$, with a standard deviation $\sigma_{g}$ rad:

$$
\left\langle e^{k}\right\rangle=0,\left\langle e^{k}, e_{k^{\prime}}\right\rangle=\sigma_{g}^{2} \delta_{k^{\prime}}^{k},\left\langle c^{i} e_{k}\right\rangle=0,
$$

then, instead of (4), from (3) follows

$$
\left\langle\varepsilon^{2}\right\rangle=\left\langle c^{i} c_{i}\right\rangle-2\left\langle c^{j} c_{i}\right\rangle h_{j}^{i}+h_{i_{1}}^{j}\left\langle c^{i_{1}} c_{i_{2}}\right\rangle h_{j}^{i_{2}}+l_{k_{1}}^{j} l_{j}^{k_{2}} \sigma_{g}^{2},
$$

and we name

$$
\gamma_{\text {meas }}^{2}=l_{k_{1}}^{j} l_{j}^{k_{2}} \sigma_{g}^{2}
$$

the measurement error term.

The jitter error appears because the subapertures centers $r_{k}$ are known only with some finite accuracy. According to (10), this leads to non-linear changes in matrix G. Usually, the uncertainty is much smaller then the size of the subapertures, and the error is negligible or can be considered as a part of the measurement error. Moreover, in this article we compare masks with regular structures with ones with irregular pattern, for which the jitter error is irrelevant. 


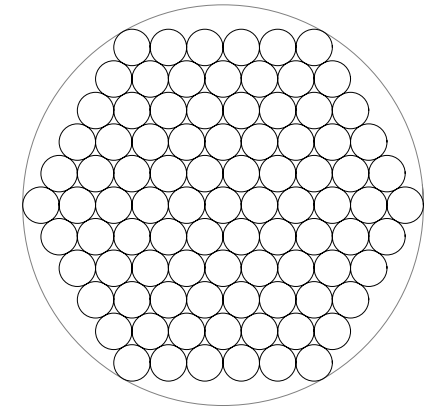

(a) $\operatorname{Hex} 91$

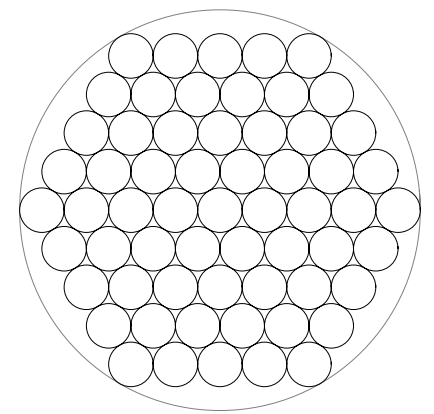

(b) Hex61

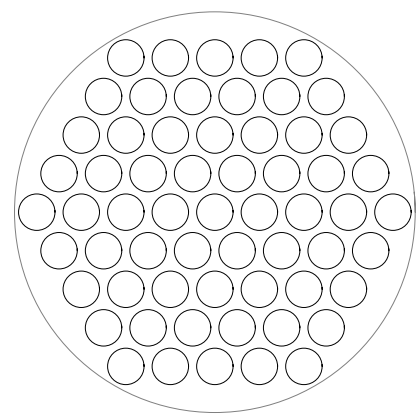

(c) Hex61s

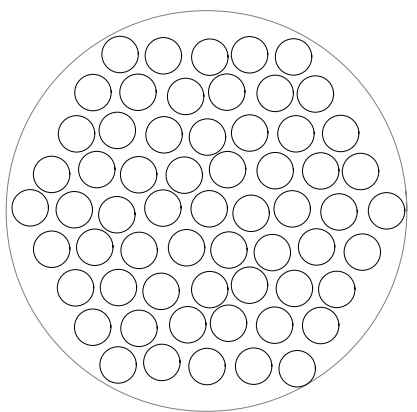

(d) move61

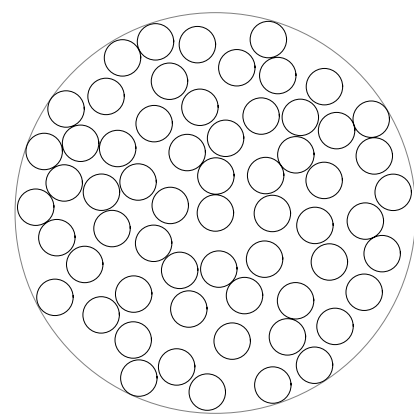

(e) MC61

Figure 2: 5 masks used for calculation of $\mathbf{H}$, shown in the unit circles. The subapertures are circles with radius $\frac{1}{11}$, except (b) with radius $\frac{1}{9}$. Each mask contains 61 subaperture, except (a) with 91 holes.

\section{IRREGULAR HS MASKS}

To investigate the dependence of the error on the mask regularity, we will calculate the matrices $\mathbf{H}$ for 5 different HS masks shown in Figure 2. Masks Hex91 and Hex61 are regular hexagonal masks with 91 and 61 subapertures (s/a) of maximum size $(1 / 11$ and $1 / 9$, resp.). Because the hexagonal mask is the densest one, the s/a size should be decreased to allow some randomisation. We have chosen to use the size of Hex91 for masks with 61 holes. Hex61s is a regular hexagonal grid with smaller s/a, move61 and MC61 represents two irregular masks.

Move61 represents a "small-movement" randomisation of the Hex61s. Each of the centres of s/a in Hex61s was moved by a random vector uniformly distributed in the circle of radius $1 / 9-1 / 11=2 / 99$ to avoid s/a overlapping. This kind of randomisation seems to be a usual approach in randomising regular structures. For instance, s/a centres of modified HS sensors considered by Dai ${ }^{9}$ can be regarded as small-movement modification.

MC61 is a more random centres distribution. We obtained it by Monte-Carlo method, by adding a random vector uniformly distributed in a circle with radius $1-1 / 11$ as a new s/a centre, if it was not closer than $2 / 11$ to any of the existing centres (to avoid overlapping) and not further than $3 / 10$ at least from one of the existing centres (to avoid too spare pattern containing less than 61 point).

For 61 s/a, the maximum possible rank of $\mathbf{G}$ is 122 . Consider a determinant of the first 122 rows of $\mathbf{G}$ as a continuous function of s/a centres $\mathbf{r}_{k}$. Then due to continuous dependence one can expect this determinant to be ill-defined for move61, and similar matrix $\mathbf{H}$. Again, looking at the Fourier transforms of the s/a centres of different types of mask, we see that small-movent randomisation has still a regular spectrum, while Monte-Carlo centres does not have any regular pattern in its transform (see Figure 3 on the next page). Thus one can expect bigger rank of $\mathbf{G}$ for Monte-Carlo mask geometry. 


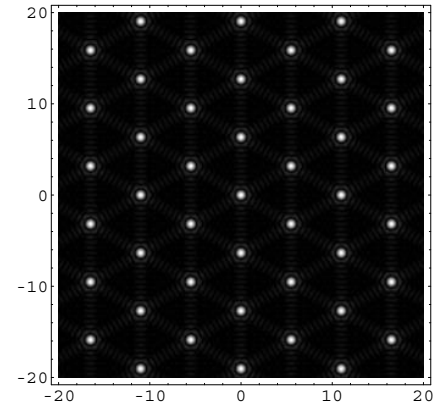

(a) Hex61

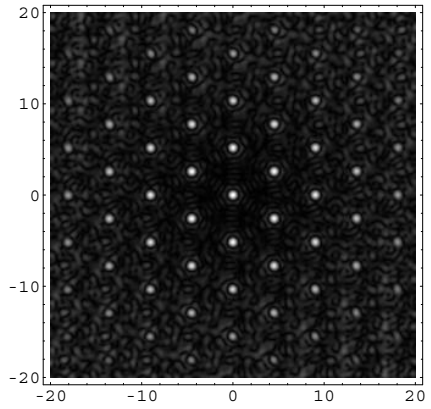

(b) move61

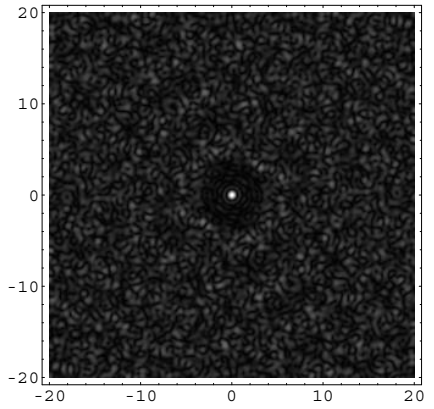

(c) MC61

Figure 3: Fourier transforms of the s/a centres for Hex61, move61, and MC61 masks.

Table 1: Properties of the HS sensing for various mask types

\begin{tabular}{lccccc}
\hline & Hex91 & Hex61 & Hex61c & move61 & MC61 \\
\hline Geometry & regular & regular & regular & small movements & Monte-Carlo \\
Number of s/a & 91 & 61 & 61 & 61 & 61 \\
Radius of s/a & $\frac{1}{11}$ & $\frac{1}{9}$ & $\frac{1}{11}$ & $\frac{1}{11}$ & $\frac{1}{11}$ \\
$N^{\prime}$ & 86 & 62 & 62 & 122 & 122 \\
$N_{\text {Opt }}^{2}$ & 29 & 29 & 23 & 23 & 42 \\
$\sigma_{\text {tr }}^{2}+\sigma_{\mathrm{al}}^{2}, \mathrm{rad}^{2}$, & & & & & 1.17998 \\
for $D=2 \mathrm{~m}, r_{0}=20 \mathrm{~cm}$ & 0.946536 & 1.17109 & 1.0295 & 0.894046 \\
\hline
\end{tabular}

\section{CALCUlation RESUlts}

We have used MATHEMATICA for our calculations. First we calculated first 1000 of Zernike polynomials $g_{i}$ in Noll's ${ }^{18}$ form using a free package written by Dr. Brett Patterson. ${ }^{19}$ To calculated the filtered basis $\frac{1}{S_{a}} \chi_{a} * \nabla g_{i}$ in analytical form for any s/a radius $a$ and s/a centre $\mathbf{r}_{k}$, we converted $g_{i}$ in polynomials in $x$ and $y$ with the origin translated in $\mathbf{r}_{k}$, and replaced every monomial $x^{i} y^{j}$ by its integral over s/a:

$$
x^{i} y^{j} \mapsto \frac{1}{\pi a^{2}} \int_{x^{2}+y^{2} \leq a^{2}} x^{i} y^{j} d x d y=\left\{\begin{array}{l}
\frac{a^{i+j} \Gamma\left(\frac{i+1}{2}\right) \Gamma\left(\frac{j+1}{2}\right)}{\pi \Gamma\left(\frac{i+j}{2}+2\right)}, \quad i \text { and } j \text { are even, } \\
0, \text { otherwise. }
\end{array}\right.
$$

Then we calculated numerically matrices $\mathbf{G}$ for 5 masks shown in Figure 2, and obtained maximum number $N^{\prime}$ of first linearly independent rows of $\mathbf{G}$ for each of the sensor configurations. The results are shown in Table 1.

From $\mathbf{G}$ we can obtain $\mathbf{H}$ for any number $N$ of decomposition modes using the Least-Squares approach given by equation (11). Figure 4 presents obtained matrices for $N=45$ and $N=122$. One can notice that for $N=45$ matrix $\mathbf{H}$ for MC61 mask contains smaller elements, while regular masks contain less non-zero elements. For maximum possible number of decomposition modes $N=122, \mathbf{H}$ for regular masks do not satisfy condition (7), and even low-order aberrations are sensed as some combination with high-order ones. This potentially could result in bigger aliasing error, as correlation coefficients usually are greater for low-order functions.

For calculation of the residual error, the turbulence statistics from Roddier's article ${ }^{20}$ was used. For number of decomposition modes less then $N^{\prime}$, the formula (8) can be used ${ }^{\dagger}$. This expression involves infinite sums, which should be

\footnotetext{
${ }^{\dagger}$ for $N>N^{\prime},(8)$ is not valid, and the variance of the error is given by (4). The plot of $\sigma_{\mathrm{al}}^{2}$ according to (9) for $N$ covering the whole range from 1 to $2 m$ has a discontinuity in monotonic behaviour in $N^{\prime}$. This allows to calculate implicitly $N^{\prime}$ for a given mask.
} 


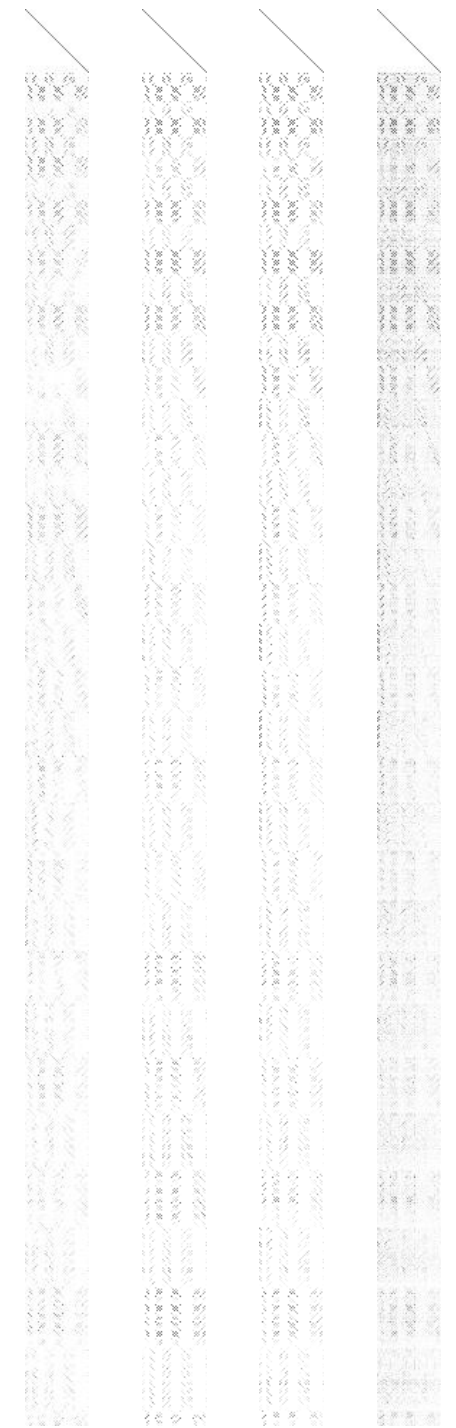

(a) (b)

Hex91 Hex61 (c)

(d)

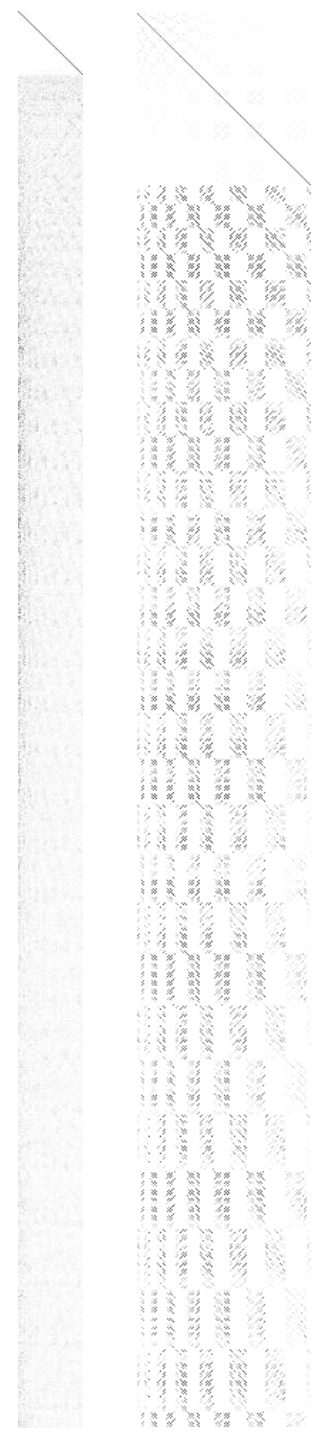

(e) (f) $\operatorname{Hex} 91$

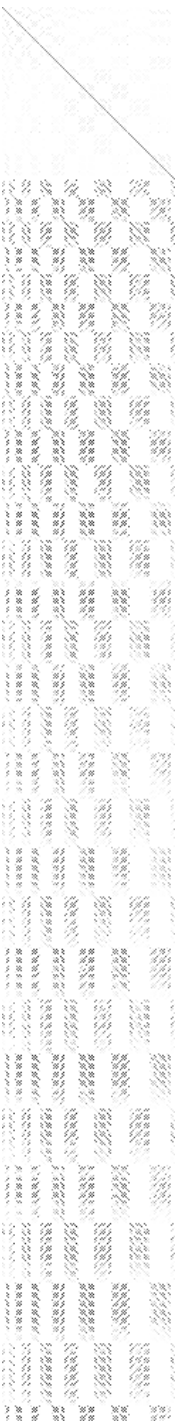

(g) Hex61

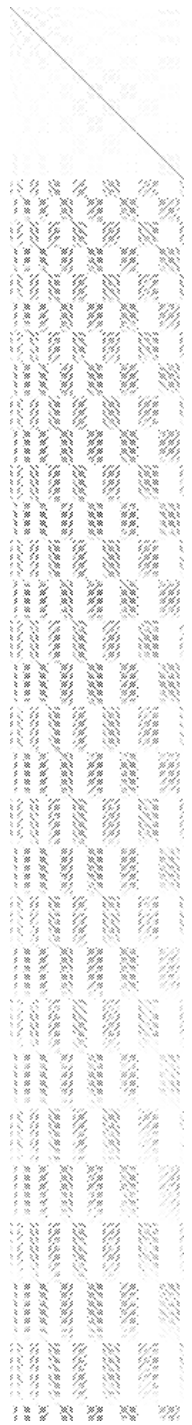

(h) Hex61s

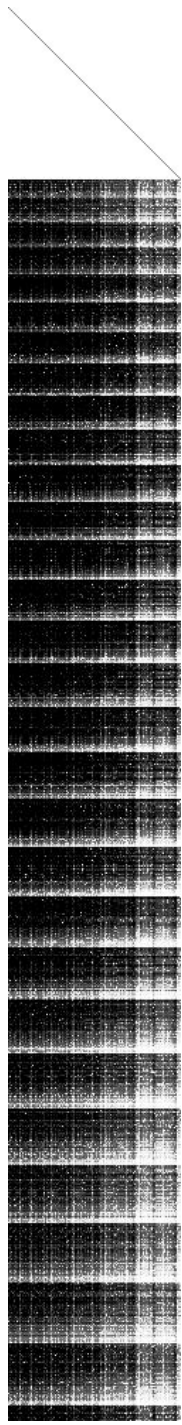

(i) move61

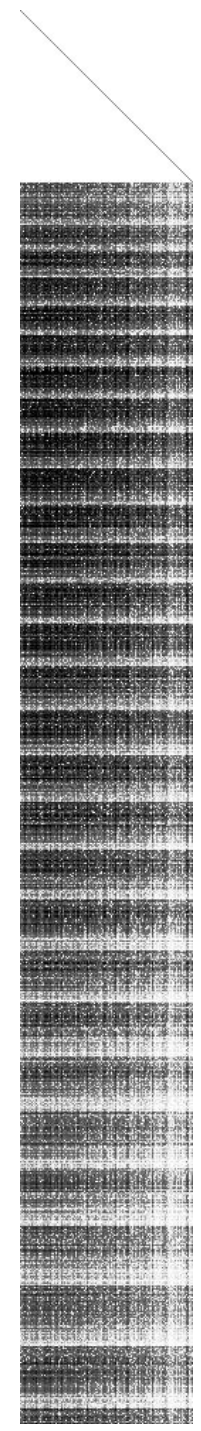

(j) MC61

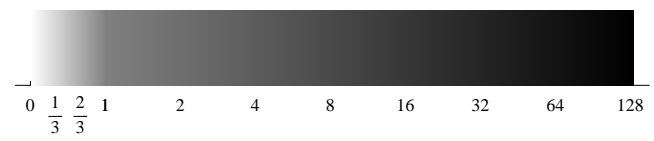

(k) Gray-scale coding

Figure 4: Matrices $\mathbf{H}$, obtained for various masks for $N=45((\mathrm{a})-(\mathrm{e}))$ and $N=122((\mathrm{f})-(\mathrm{j}))$ decomposition modes. Absolute values of non-zero elements are indicated by a gray level scale shown in Fig. (k). 


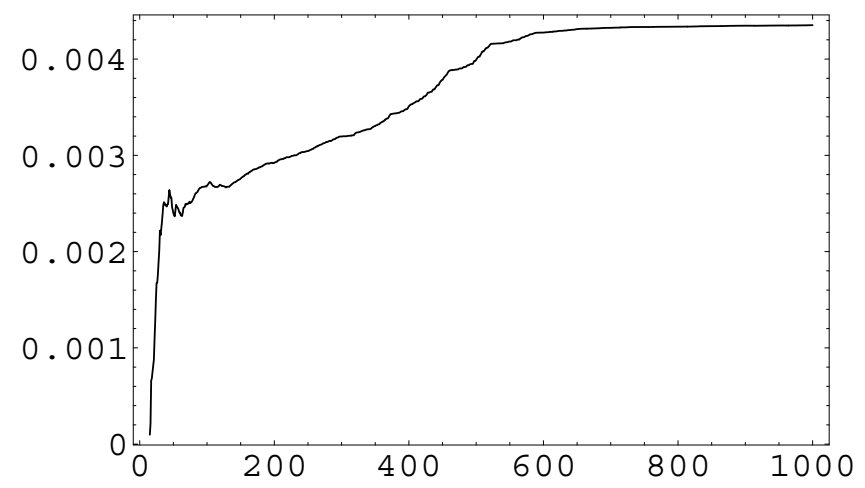

Figure 5: An infinite sum $h_{l_{1}}^{j}\left\langle c^{l_{1}} c_{l_{2}}\right\rangle h_{j}^{l_{2}}$ for $\mathbf{H}_{\text {move61 }}$ for $N=15$ decomposition modes is approximated by a finite one with $l_{1}, l_{2}=N+1, \ldots, L$ and plotted against $L$.

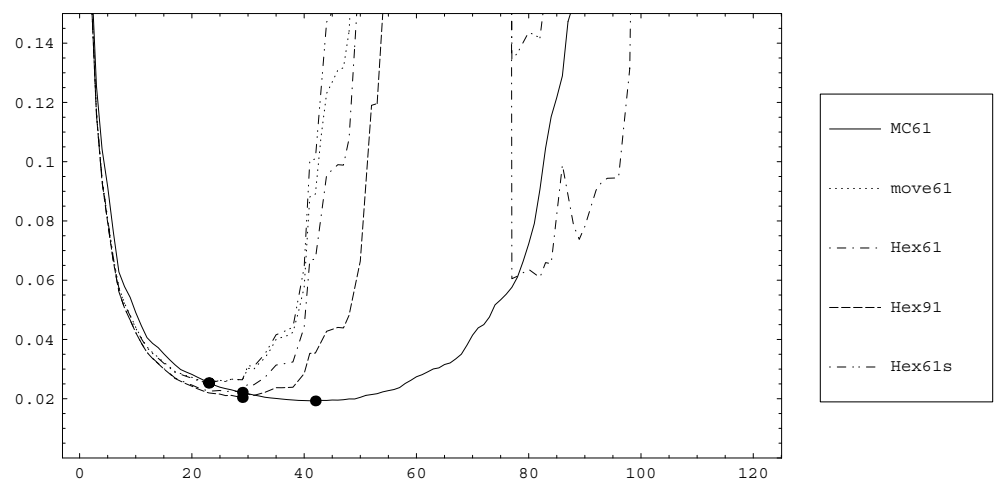

Figure 6: Sum of aliasing and truncation errors, in $\left(D / r_{0}\right)^{\frac{5}{3}}$.

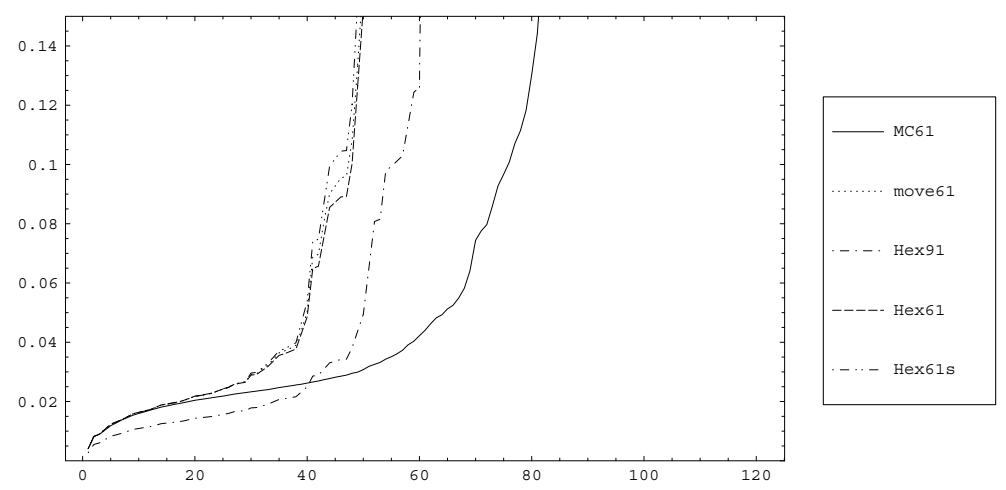

Figure 7: Measurement error, in $\sigma_{g}^{2}$. 
approximated for practical purposes. We used Dai's ${ }^{9}$ asymptotical approximation for the infinite-series truncation term $\left\langle c^{l} c_{l}\right\rangle$,

$$
\left\langle c^{l} c_{l}\right\rangle \asymp 0.274 N^{-0.8428}\left(\frac{D}{r_{0}}\right)^{5 / 3},
$$

and approximated infinite sum in the aliasing term $h_{l_{1}}^{j}\left\langle c^{l 1} c_{l_{2}}\right\rangle h_{j}^{l_{2}}$ by a finite one, letting $l_{1}, l_{2}$ to change from $N+1$ to some $L$. For matrices $\mathbf{H}$ under consideration, the aliasing term can be considered to be a constant for $L>700$ (see Figure 5 for an illustration), and thus we used $L=700$ in our calculations.

The results for the contribution of aliasing and series truncation terms in the residual error are presented in Figure 6. The plot presents the variance of truncation and aliasing errors (in $\left(\frac{D}{r_{0}}\right)^{5 / 3} \mathrm{rad}^{2}$ ) against the number of decomposition modes $N$. As it was expected, the Monte-Carlo randomisation of the s/a centres results in a smaller error and in the same time can be used for a bigger number of decomposition modes. Table 1 shows optimal number of decomposition modes $N_{\text {Opt }}$ for all of the masks and minimum aliasing plus truncation error calculated for $\left(D / r_{0}\right)=10$.

The measurement error was calculated for each mask by (12) in terms of slopes measurement variance $\sigma_{g}^{2}$, which depends strongly on the application parameters, such as detector sensitivity and pixel size, spectrum of the light, etc. Added to the truncation and aliasing error, the measurement error can affect the optimal number of decomposition modes and, of course, the total restoration error. Nevertheless, from Figure 7 it follows that the total error still be the smallest for mask MC61 for $N>40$.

\section{DISCUSSION AND CONCLUSIONS}

In this paper we have developed the reasoning of Dai ${ }^{11}$ to the general case of modal wavefront reconstruction. We have shown, that independently on the choice of the native wavefront basis and reconstruction function set, the modal wavefront reconstruction with HS wavefront sensor can be presented as linear operator in space of continuous functions. As this operator maps infinite-dimensional space into finite-dimensional one, there is an infinite-dimensional space of function reconstructed as zero by the sensor. As a consequence, no statement about the accuracy of the HS-sensor-based measurements should be made in the lack of statistical model of the incoming wavefront.

We have also illustrated the developed model by calculating the total error of wavefront reconstruction with Zernike polynomials, in the case of Kolmogorov's turbulence. The error was calculated for various geometry of the sensor subapertures array. According to the calculations, the use of 61-holes mask with randomly distributed centres gives better results then the regular hexagonal mask with 91 subapertures of the same size.

The results can be different for another wavefront statistics, but comparing of the matrices for Zernike basis for instance, for 45 decomposition modes allows to expect better performance of the random masks. This encourages to use randomized masks in practice.

Some expected problems with location of the spot centroids due to the irregular nature of the mask are related with the need of calibration, which, in principle, increases the slope measurement error. But in practice, any Hartmann sensor is calibrated first, and thus the slope measurement error increases equally for regular and irregular mask. Associated measurement error of the Hartmann sensor, as was shown, is smaller for the irregular masks starting from some number of decomposition modes.

The proposed model allows also to estimate the error of the correction with active element's influence functions, which are usually not orthogonal. In this case, the use of irregular masks was not yet justified. Also an interesting problem in this case is the construction of the optimal decomposition operator $\mathcal{L}$, either for a given statistics or for least-square approximation. This makes the aim for our future research.

\section{ACKNOWLEDGMENTS}

The authors are very grateful to Dr. Alexey Simonov for fruitful discussions that contributed a lot in understanding of theoretical and practical aspects of the problem. 


\section{REFERENCES}

1. I. Ghozeil, Optical Shop Testing, ch. Hartmann and Other Screen Tests, pp. 367 - 396. John Wiley \& Sons, Inc., 2nd ed., 1992.

2. R. K. Tyson, Principles of adaptive opticsrieg, Academic Press, 2nd ed., 1998.

3. D. L. Fried, "Least-squares fitting a wave-front distortion estimate to an array of phase-difference measurements," $J$. Opt. Soc. Am. 67, pp. 370 - 375, Mar. 1977.

4. B. R. Hunt, "Matrix formulation of the reconstruction of phase values from phase differences," J. Opt. Soc. Am. 69, pp. 393 -399, Mar. 1979.

5. W. H. Southwell, "Wave-front estimation from wave-front slope measurements," J. Opt. Soc. Am. 70, pp. 998 - 1006, apr 1980.

6. K. R. Freischlad and C. L. Koliopoulos, "Modal estimation of a wave front from difference measurements using the discrete fourier transform," J. Opt. Soc. Am. A 3, pp. 1852 - 1861, Nov. 1986.

7. M. C. Roggemann, "Optical perfomance of fully and partially compensated adaptive optics systems using leastsquares and minimum variance phase reconstructors," Computers Elect. Engng 18(6), pp. 451-466, 1992.

8. R. G. Lane and M. Tallon, "Wavefront reconstruction using a shack-hartmann sensor," Appl. Opt. 31, pp. $6902-$ 6908, Nov. 1992.

9. G.-m. Dai, "Modified Hartmann-Shack wavefront sensing and iterative wavefront reconstruction," in Adaptive Optics in Astronomy, Proceedings of SPIE 2201, pp. 562 - 573, SPIE, 1994.

10. G.-m. Dai, "Modal compensation of atmospheric turbulence with the use of Zernike polynomials and KarhunenLoève functions," J. Opt. Soc. Am. A 12, pp. 2182 - 2193, Oct. 1995.

11. G.-m. Dai, "Modal wavefront reconstruction with Zernike polynomials and Karhunen-Loève functions," J. Opt. Soc. Am. A 13, pp. 1218-1225, jun 1996.

12. M. C. Roggemann and T. J. Schulz, "Algorithm to increase the largest aberration that can be reconstructed from hartmann sensor measurements," Appl. Opt. 37, pp. 4321-4329, July 1998.

13. W. Zou and Z. Zhang, "Generalized wave-front reconstruction algorithm applied in a shack-hartmann test," Appl. Opt. 39, pp. 250 - 268, Jan. 2000.

14. Y. Carmon and E. N. Ribak, "Phase retrieval by demodulation of a hartmann-shack sensor," Optics Communications 215, pp. 285-288, Jan. 2003.

15. L. A. Poyneer and J.-P. Véran, "Optimal modal fourier-transform wavefront control," J. Opt. Soc. Am. A 22, pp. 1515 - 1526, Aug. 2005.

16. A. Talmi and E. N. Ribak, "Direct demodulation of hartmann-shack patterns," J. Opt. Soc. Am. A 21, pp. 632 - 639, Apr. 2004.

17. J. Herrmann, "Cross coupling and aliasing in modal wave-front estimation," J. Opt. Soc. Am. 71, pp. 989-992, Aug 1981.

18. R. J. Noll, “Zernike polynomials and atmospheric turbulence,” J. Opt. Soc. Am. 66, pp. 207 - 211, Mar. 1976.

19. B. Patterson, "Circular and annular Zernike polynomials; MATHEMATICA package." http: / / i ibrary . wolfram . com/infocenter/MathSource/4483/, Mar. 2002. UK Astronomy Technology Centre.

20. N. Roddier, "Atmospheric wavefront simulation using Zernike polynomials," Optical Engineering 29, pp. 1174 1180, Oct. 1990. 